\title{
КОЛМОГОРОВСКИЕ ПОПЕРЕЧНИКИ КЛАССОВ СОБОЛЕВА НА ОТРЕЗКЕ С ОГРАНИЧЕНИЯМИ НА ВАРИАЦИЮ ${ }^{1}$
}

\author{
А. А. Васильева
}

\begin{abstract}
В работе исследуется задача о колмогоровских поперечниках в пространстве $L_{q}[0,1]$ классов Липшица на отрезке с фиксированными значениями в нескольких точках: $\tilde{M}=\left\{f \in A C[0,1],\|\dot{f}\|_{\infty} \leq 1, f(j / s)=\right.$ $\left.y_{j}, 0 \leq j \leq s\right\}$. Из известных результатов о поперечниках классов Соболева легко получить порядковые оценки с точностью до констант, зависящих от $q$ и $y_{1}, \ldots, y_{n}$. Здесь получены порядковые оценки с точностью до констант, зависящих только от $q$. Задача сводится к оценке поперечников пересечения двух конечномерных множеств: куба и произведения октаэдров с некоторыми весами. Если заменить куб на шар пространства $l_{p}^{n}$, то получается дискретизация задачи о поперечнике пересечения класса Соболева и класса функций с ограничениями на вариацию: $M=\left\{f \in A C[0,1]:\|\dot{f}\|_{L_{p}[0,1]} \leq 1,\|\dot{f}\|_{L_{1}[(j-1) / s, j / s]} \leq\right.$ $\left.\varepsilon_{j} / s, \quad 1 \leq j \leq s\right\}$. Для достаточно больших $n$ получены порядковые оценки поперечников этих классов с точностью до констант, зависящих только от $p$ и $q$. Оказывается, что если $p>q$ или $p>2$, то эти оценки имеют вид $\varphi\left(\varepsilon_{1}, \ldots, \varepsilon_{s}\right) n^{-1}$, где $\varphi\left(\varepsilon_{1}, \ldots, \varepsilon_{s}\right) \rightarrow 0$ при $\left(\varepsilon_{1}, \ldots, \varepsilon_{s}\right) \rightarrow 0$ (явные формулы для $\varphi$ приведены в тексте статьи). Если $p \leq 2$ и $p \leq q$, то оценки имеют вид $n^{-1}$ (то есть ограничения на вариацию оценку поперечников при больших $n$ не улучшают). Для доказательства оценок сверху используется результат Э. М. Галеева о пересечении конечномерных шаров. Для доказательства оценок снизу обобщается результат Е.Д. Глускина о поперечнике пересечения куба и октаэдра.
\end{abstract}

Ключевые слова: поперечники по Колмогорову, классы Соболева, интерполяционные классы.

A. A. Vasil'eva. Kolmogorov widths of Sobolev classes on a closed interval with constraints on the variation.

We study the problem of estimating Kolmogorov widths in $L_{q}[0,1]$ for the Lipschitz classes of functions with fixed values at several points: $\tilde{M}=\left\{f \in A C[0,1],\|\dot{f}\|_{\infty} \leq 1, f(j / s)=y_{j}, 0 \leq j \leq s\right\}$. Applying well-known results about the widths of Sobolev classes, it is easy to obtain order estimates up to constants depending on $q$ and $y_{1}, \ldots, y_{n}$. Here we obtain order estimates up to constants depending only on $q$. To this end, we estimate the widths of the intersection of two finite-dimensional sets: a cube and a weighted Cartesian product of octahedra. If we take the unit ball of $l_{p}^{n}$ instead of the cube, we get a discretization of the problem on estimating the widths of the intersection of the Sobolev class and the class of functions with constraints on their variation: $M=\left\{f \in A C[0,1]:\|\dot{f}\|_{L_{p}[0,1]} \leq 1,\|\dot{f}\|_{L_{1}[(j-1) / s, j / s]} \leq \varepsilon_{j} / s, 1 \leq j \leq s\right\}$. For sufficiently large $n$, order estimates are obtained for the widths of these classes up to constants depending only on $p$ and $q$. If $p>q$ or $p>2$, then these estimates have the form $\varphi\left(\varepsilon_{1}, \ldots, \varepsilon_{s}\right) n^{-1}$, where $\varphi\left(\varepsilon_{1}, \ldots, \varepsilon_{s}\right) \rightarrow 0$ as $\left(\varepsilon_{1}, \ldots, \varepsilon_{s}\right) \rightarrow 0$ (explicit formulas for $\varphi$ are given in the paper). If $p \leq q$ and $p \leq 2$, then the estimates have the form $n^{-1}$ (hence, the constraints on the variation do not improve the estimate for the widths). The upper estimates are proved with the use of Galeev's result on the intersection of finite-dimensional balls, whereas the proof of the lower estimates is based on a generalization of Gluskin's result on the width of the intersection of a cube and an octahedron.

Keywords: Kolmogorov widths, Sobolev classes, interpolation classes.

MSC: $41 \mathrm{~A} 46$

DOI: $10.21538 / 0134-4889-2019-25-2-48-66$

\section{Введение}

В работе [1] изучалась задача о непустоте множества функций $M_{0}=\left\{f \in C^{1}[a, b]: f_{1}(t) \leq\right.$ $\left.f(t) \leq f_{2}(t), g_{1}(t) \leq \dot{f}(t) \leq g_{2}(t)\right\}$, где $f_{1}, f_{2}, g_{1}, g_{2}:[a, b] \rightarrow \mathbb{R} \cup\{ \pm \infty\}$. Позже И. Г. Царьковым была поставлена задача об оценках колмогоровских поперечников таких классов функций в пространстве Лебега. Здесь эта задача будет исследоваться в частном случае:

$$
M_{0}=\left\{f \in C^{1}[0,1]: \forall t \in[0,1]|\dot{f}(t)| \leq 1, \quad f\left(\frac{j}{s}\right)=y_{j}, \quad 0 \leq j \leq s\right\},
$$

\footnotetext{
${ }^{1}$ Работа выполнена при поддержке РФФИ (проект 19-01-00332).
} 
где $y_{0}, \ldots, y_{s}$ - заданные числа. Напомним, что колмогоровский поперечник порядка $n \in \mathbb{Z}_{+}$ подмножества в линейном нормированном пространстве $X$ определяется как

$$
d_{n}(C, X)=\inf _{L \in \mathcal{L}_{n}(X)} \sup _{x \in C} \inf _{y \in L}\|x-y\|,
$$

где $\mathcal{L}_{n}(X)$ - совокупность подпространств в $X$ размерности не выше $n$ (см. [2-4]).

Если класс $M_{0}$ непуст и не состоит из одной функции, то из оценок поперечников классов липшицевых функций следует, что

$$
C n^{-1} \leq d_{n}\left(M_{0}, L_{q}[0,1]\right) \leq n^{-1},
$$

где $C>0$ зависит от $y_{0}, \ldots, y_{s}$. Здесь будут получены при достаточно больших $n \in \mathbb{N}$ порядковые оценки поперечников $d_{n}\left(M_{0}, L_{q}[0,1]\right)$ с точностью до констант, не зависящих от $y_{0}, \ldots, y_{s}$.

Отметим, что у множества

$$
\tilde{M}=\left\{f \in A C[0,1]:|\dot{f}(t)| \leq 1 \text { п.в., } \quad f\left(\frac{j}{s}\right)=y_{j}, \quad 0 \leq j \leq s\right\}
$$

поперечники будут такими же, как у $M_{0}$.

С помощью метода дискретизации задача сводится к оценке поперечников пересечения конечномерных шаров: шара в $l_{\infty}^{N}$ с некоторым весом и декартова произведения многомерных октаэдров разных размеров (подробнее об этом будет сказано в следующем разделе). Задача об оценке поперечников пересечений и произведений конечномерных шаров изучалась в работах Э. М. Галеева [5-8], Е. Д. Глускина [9], А. Д. Изаака [10;11], Ю. В. Малыхина, К. С. Рютина [12] и др.

Если вместо шара в весовом $l_{\infty}^{N}$ рассмотреть шар в весовом $l_{p}^{N}$, то получится задача, возникающая при дискретизации задачи об оценке поперечника класса

$$
M=\left\{f \in A C[0,1]:\|\dot{f}\|_{L_{p}[0,1]} \leq 1, \quad\|\dot{f}\|_{L_{1}\left[\frac{j-1}{s}, \frac{j}{s}\right]} \leq \frac{\varepsilon_{j}}{s}, \quad 1 \leq j \leq s\right\},
$$

где $\varepsilon_{j} \in[0,1], 1 \leq j \leq n,-$ некоторые заданные числа.

В данной работе будут получены при достаточно больших $n \in \mathbb{N}$ порядковые оценки колмогоровских поперечников классов $M$ и $\tilde{M}$ в пространстве $L_{q}[0,1]$ с точностью до мультипликативных констант, зависящих только от $p$ и $q$.

Мы будем использовать следующее обозначение для порядковых неравенств. Пусть $X, Y-$

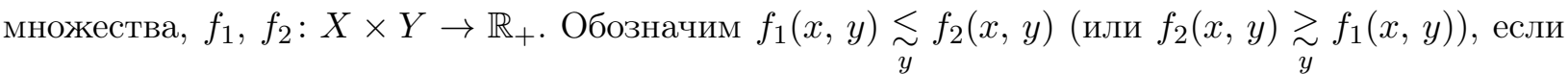
для любого $y \in Y$ существует $c(y)>0$ такое, что $f_{1}(x, y) \leq c(y) f_{2}(x, y)$ для любого $x \in X$; $f_{1}(x, y) \underset{y}{\asymp} f_{2}(x, y)$, если $f_{1}(x, y) \underset{y}{\lesssim} f_{2}(x, y)$ и $f_{2}(x, y) \underset{y}{\lesssim} f_{1}(x, y)$.

Сформулируем основные результаты работы.

Теорема 1. Пусть $1<p<\infty, 1<q<\infty, s \in \mathbb{N}, \varepsilon_{j} \in[0,1], 1 \leq j \leq s$.

1. Пусть $p>2, q \geq 2$. Положим $\theta=\frac{q+2}{q} \frac{p-1}{p-2}$. Тогда при достаточно больших $n \in \mathbb{N}$

$$
d_{n}\left(M, L_{q}[0,1]\right) \underset{p, q}{\asymp} n^{-1}\left(\sum_{j=1}^{s} \frac{\varepsilon_{j}^{\frac{1}{\theta+1}}}{s}\right)^{(\theta+1) \frac{p-2}{2(p-1)}} .
$$

Пусть $p>q, q<2$. Положим $\theta=\frac{q(p-1)}{p-q}$. Тогда при достаточно больших $n \in \mathbb{N}$

$$
d_{n}\left(M, L_{q}[0,1]\right) \underset{p, q}{\asymp} n^{-1}\left(\sum_{j=1}^{s} \frac{\varepsilon_{j}^{\frac{1}{\theta+1}}}{s}\right)^{(\theta+1) \frac{p-q}{q(p-1)}} .
$$


2. Пусть $p \leq q, p \leq 2$. Тогда при достаточно больших $n \in \mathbb{N}$

$$
d_{n}\left(M, L_{q}[0,1]\right) \underset{p, q}{\asymp} n^{-\max \left\{1+\frac{1}{q}-\frac{1}{p}, 1+\frac{1}{2}-\frac{1}{p}\right\}} .
$$

Теперь рассмотрим класс $\tilde{M}$. Его непустота равносильна условию $\left|y_{j}-y_{j-1}\right| \leq \frac{1}{s}, 1 \leq j \leq s$. Положим

$$
\varepsilon_{j}=1-s\left|y_{j}-y_{j-1}\right| .
$$

Теорема 2. Пусть $1<q<\infty, s \in \mathbb{N}, \varepsilon_{j} \in[0,1]$ заданы формулой $(0.3), 1 \leq j \leq s$. Положим $\theta=\frac{q+2}{q}$ при $q \geq 2, \theta=q$ при $q<2$. Тогда

$$
d_{n}\left(\tilde{M}, L_{q}[0,1]\right) \asymp \begin{cases}n^{-1}\left(\sum_{j=1}^{s} \frac{\varepsilon_{j}^{\frac{1}{\theta+1}}}{s}\right)^{\frac{\theta+1}{2}}, & q \geq 2, \\ n^{-1}\left(\sum_{j=1}^{s} \frac{\varepsilon_{j}^{\frac{1}{\theta+1}}}{s}\right)^{\frac{\theta+1}{q}}, & q<2 .\end{cases}
$$

В разд. 2 задачи о поперечниках классов $M$ и $\tilde{M}$ с помощью метода дискретизации сводятся к оценкам поперечников конечномерных множеств. В разд. 3 доказываются оценки сверху поперечников этих множеств. В разд. 4 доказываются оценки снизу.

\section{1. Дискретизация задач}

Через $l_{p}^{\nu}$ обозначаем пространство $\mathbb{R}^{\nu}$ с нормой

$$
\left\|\left(x_{1}, \ldots, x_{\nu}\right)\right\|_{l_{p}^{\nu}}^{p}=\sum_{i=1}^{\nu}\left|x_{i}\right|^{p}
$$

Сначала рассмотрим задачу об оценке поперечников класса $M$.

Пусть $\nu_{j} \in \mathbb{N}, 1 \leq j \leq s$ (их мы подберем позже), $m \in \mathbb{Z}_{+}$. Разобьем каждый из отрезков $\left[\frac{j-1}{s}, \frac{j}{s}\right]$ на $2^{m} \nu_{j}$ одинаковых отрезков $\Delta_{j, i}^{m}=\left[t_{i-1, j}^{m}, t_{i, j}^{m}\right], 1 \leq i \leq 2^{m} \nu_{j}$. Определим функции $\varphi_{i, j}^{m}$ по формуле

$$
\varphi_{i, j}^{m}=\varkappa_{j}^{m} \max \left\{\frac{t_{i, j}^{m}-t_{i-1, j}^{m}}{2}-\left|t-\frac{t_{i-1, j}^{m}+t_{i, j}^{m}}{2}\right|, 0\right\},
$$

где $\varkappa_{j}^{m}>0$ выбирается так, что $\left\|\varphi_{i, j}^{m}\right\|_{L_{q}[0,1]}=1$. Тогда

$$
\varphi_{i, j}^{m}\left(\frac{t_{i-1, j}^{m}+t_{i, j}^{m}}{2}\right) \underset{q}{\widetilde{q}}\left(2^{m} s \nu_{j}\right)^{1 / q},
$$

и для любых $c_{j, i} \in \mathbb{R}, 1 \leq j \leq s, 1 \leq i \leq 2^{m} \nu_{j}$, выполнено

$$
\left\|\sum_{j=1}^{s} \sum_{i=1}^{2^{m} \nu_{j}} c_{j, i} \varphi_{i, j}^{m}\right\|_{L_{q}[0,1]}=\left(\sum_{j=1}^{s} \sum_{i=1}^{2^{m} \nu_{j}}\left|c_{j, i}\right|^{q}\right)^{1 / q} .
$$

Дискретизация для ощенки сверху. Отметим, что достаточно рассмотреть случаи 1 и 2 в теореме 1 , так как при $p \leq q, p \leq 2$ оценка сверху следует из оценок поперечников классов Соболева в $L_{q}[0,1]$, полученных в [13;14]. Пусть $f \in M$. Через $P_{m} f$ обозначим кусочно-линейную функцию такую, что $P_{m} f\left(t_{i, j}^{m}\right)=f\left(t_{i, j}^{m}\right), 1 \leq j \leq s, 0 \leq i \leq 2^{m} \nu_{j}$. Тогда

$$
f=P_{0} f+\sum_{m \in \mathbb{Z}_{+}}\left(P_{m+1} f-P_{m} f\right)
$$


(ряд сходится равномерно). Покажем, что

$$
\sum_{j=1}^{s}\left(2^{m} \nu_{j} s\right)^{p} \sum_{i=1}^{2^{m} \nu_{j}}\left\|P_{m+1} f-P_{m} f\right\|_{L_{p}\left(\Delta_{j, i}^{m}\right)}^{p} \underset{p}{\lesssim} 1, \quad f \in M
$$

В самом деле,

$$
\sum_{i=1}^{2^{m} \nu_{j}} \int_{\Delta_{j, i}^{m}}\left|P_{m+1} f(t)-P_{m} f(t)\right|^{p} d t \underset{p}{\lesssim} \sum_{i=1}^{2^{m} \nu_{j}}\left|\Delta_{j, i}^{m}\right|^{p}\|\dot{f}\|_{L_{p}\left(\Delta_{j, i}^{m}\right)}^{p}=\frac{1}{\left(2^{m} \nu_{j} s\right)^{p}} \int_{(j-1) / s}^{j / s}|\dot{f}|^{p} d t
$$

Умножим эти неравенства на $\left(2^{m} \nu_{j} s\right)^{p}$ и просуммируем по $j$; так как $\int_{0}^{1}|\dot{f}|^{p} d t \stackrel{(0.2)}{\leq} 1$, то получим (1.4).

Отметим, что существуют числа $c_{j, i}^{m}$ такие, что

$$
P_{m+1} f-P_{m} f=\sum_{j=1}^{s} \sum_{i=1}^{2^{m} \nu_{j}} c_{j, i}^{m} \varphi_{i, j}^{m}
$$

Так как у функций $\varphi_{i, j}^{m}$ носители не перекрываются, то из $(1.4),(1.5)$ следует, что

$$
\sum_{j=1}^{s}\left(2^{m} \nu_{j} s\right)^{p} \sum_{i=1}^{2^{m} \nu_{j}}\left|c_{j, i}^{m}\right|^{p}\left\|\varphi_{i, j}^{m}\right\|_{L_{p}\left(\Delta_{j, i}^{m}\right)}^{p} \underset{p}{\lesssim} 1
$$

Так как $\left\|\varphi_{i, j}^{m}\right\|_{L_{p}\left(\Delta_{j, i}^{m}\right)} \underset{p}{ }\left|\Delta_{j, i}^{m}\right|^{1 / p}\left\|\varphi_{i, j}^{m}\right\|_{C\left(\Delta_{j, i}^{m}\right)} \underset{p, q}{(.1)}\left(2^{m} s \nu_{j}\right)^{\frac{1}{q}-\frac{1}{p}}$, то для $f \in M$ выполнено

$$
\sum_{j=1}^{s}\left(2^{m} \nu_{j} s\right)\left(1+\frac{1}{q}-\frac{1}{p}\right) p \sum_{i=1}^{2^{m} \nu_{j}}\left|c_{j, i}^{m}\right|^{p} \underset{p, q}{\lesssim} 1
$$

Положим $\tau_{i, j}^{m}=\frac{t_{i-1, j}^{m}+t_{i, j}^{m}}{2}$. Тогда

$$
\sum_{i=1}^{2^{m} \nu_{j}}\left(\left|f\left(t_{i-1, j}^{m}\right)-f\left(\tau_{i, j}^{m}\right)\right|+\left|f\left(\tau_{i, j}^{m}\right)-f\left(t_{i, j}^{m}\right)\right|\right) \leq \sum_{i=1}^{2^{m} \nu_{j}} \int_{t_{i-1, j}^{m}}^{t_{i, j}^{m}}|\dot{f}(t)| d t \stackrel{(0.2)}{\leq} \frac{\varepsilon_{j}}{s}
$$

Для $t \in \Delta_{j, i}^{m}$ выполнено $P_{m+1} f(t) \stackrel{(1.5)}{=} P_{m} f(t)+c_{j, i}^{m} \varphi_{i, j}^{m}(t)$. Поэтому

$$
\begin{gathered}
\left|c_{j, i}^{m} \varphi_{i, j}^{m}\left(\tau_{i, j}^{m}\right)\right|=\left|P_{m+1} f\left(\tau_{i, j}^{m}\right)-P_{m} f\left(\tau_{i, j}^{m}\right)\right|=\left|P_{m+1} f\left(\tau_{i, j}^{m}\right)-\frac{P_{m} f\left(t_{i-1, j}^{m}\right)+P_{m} f\left(t_{i, j}^{m}\right)}{2}\right| \\
\leq\left|f\left(t_{i-1, j}^{m}\right)-f\left(\tau_{i, j}^{m}\right)\right|+\left|f\left(\tau_{i, j}^{m}\right)-f\left(t_{i, j}^{m}\right)\right| .
\end{gathered}
$$

Значит,

$$
\sum_{i=1}^{2^{m} \nu_{j}}\left|c_{j, i}^{m}\right| \cdot\left|\varphi_{i, j}^{m}\left(\tau_{i, j}^{m}\right)\right| \leq \frac{\varepsilon_{j}}{s}
$$

Таким образом,

$$
\sum_{i=1}^{2^{m} \nu_{j}}\left|c_{j, i}^{m}\right| \underset{q}{(1.1)} \frac{\varepsilon_{j}}{s} \frac{1}{\left(2^{m} \nu_{j} s\right)^{1 / q}}
$$


Пусть $N_{m}=\sum_{j=1}^{s} 2^{m} \nu_{j}, V_{m} \subset \mathbb{R}^{N_{m}}-$ множество последовательностей $\left(c_{j, i}^{m}\right)_{1 \leq j \leq s, 1 \leq i \leq 2^{m} \nu_{j}}$, удовлетворяющих

$$
\begin{gathered}
\sum_{\substack{j=1 \\
2^{m} \nu_{j}}}^{s}\left(2^{m} \nu_{j} s\right)^{\left(1+\frac{1}{q}-\frac{1}{p}\right) p} \sum_{i=1}^{2^{m} \nu_{j}}\left|c_{j, i}^{m}\right|^{p} \leq 1, \\
\sum_{i=1}\left|c_{j, i}^{m}\right| \leq \frac{\varepsilon_{j}}{s} \frac{1}{\left(2^{m} \nu_{j} s\right)^{1 / q}}, \quad 1 \leq j \leq s .
\end{gathered}
$$

Пусть $\sum_{j=1}^{s} \nu_{j}=n$. Применяя аналог метода дискретизации В. Е. Майорова [16] и учитывая $(1.2),(1.3),(1.6)$ и $(1.7)$, получаем

$$
d_{2 n+1}\left(M, L_{q}[0,1]\right) \underset{p, q}{\lesssim} \sum_{m \in \mathbb{Z}_{+}} d_{k(m)}\left(V_{m}, l_{q}^{N_{m}}\right)
$$

где $k(m) \in \mathbb{Z}_{+}, \sum_{m \in \mathbb{Z}_{+}} k(m) \leq n$. Тем самым, для оценки сверху в теореме 1 достаточно подобрать числа $\nu_{j}$ и $k(m)$ так, чтобы $\sum_{j=1}^{s} \nu_{j}=n, \sum_{m \in \mathbb{Z}_{+}} k(m) \leq n$,

$$
\sum_{m \in \mathbb{Z}_{+}} d_{k(m)}\left(V_{m}, l_{q}^{N_{m}}\right) \underset{p, q}{\lesssim} \begin{cases}n^{-1}\left(\sum_{j=1}^{s} \frac{\varepsilon_{j}^{\frac{1}{\theta+1}}}{s}\right)^{(\theta+1) \frac{p-2}{2(p-1)}}, & p>2, q \geq 2, \\ n^{-1}\left(\sum_{j=1}^{s} \frac{\varepsilon_{j}^{\frac{1}{\theta+1}}}{s}\right)^{(\theta+1) \frac{p-q}{q(p-1)}}, & p>q, q<2,\end{cases}
$$

где

$$
\theta= \begin{cases}\frac{q+2}{q} \frac{p-1}{p-2}, & p>2, q \geq 2, \\ \frac{q(p-1)}{p-q}, & p>q, q<2 .\end{cases}
$$

Это будет сделано в разд. 3.

Дискретизачия для оценки снизу. Пусть $\sum_{j=1}^{s} \nu_{j}=n, V \subset \mathbb{R}^{n}-$ пространство последовательностей $\left(c_{j, i}\right)_{1 \leq j \leq s, 1 \leq i \leq \nu_{j}}$ таких, что

$$
\begin{aligned}
& \sum_{j=1}^{s}\left(\nu_{j} s\right)^{\left(1+\frac{1}{q}-\frac{1}{p}\right) p} \sum_{i=1}^{\nu_{j}}\left|c_{j, i}\right|^{p} \leq 1, \\
& \sum_{i=1}^{\nu_{j}}\left|c_{j, i}\right| \leq \frac{\varepsilon_{j}}{s} \frac{1}{\left(\nu_{j} s\right)^{1 / q}}, \quad 1 \leq j \leq s .
\end{aligned}
$$

Пусть $f=\sum_{j=1}^{s} \sum_{i=1}^{\nu_{j}} c_{j, i} \varphi_{i, j}^{0},\left(c_{j, i}\right)_{1 \leq j \leq s, 1 \leq i \leq \nu_{j}} \in V$. Отметим, что при п.в. $t \in \Delta_{i, j}^{0}$ выполнено $\left|\dot{\varphi}_{i, j}^{0}(t)\right| \stackrel{(1.1)}{\widehat{\tau}}\left(s \nu_{j}\right)^{1+\frac{1}{q}}$. Значит,

$$
\begin{aligned}
& \|\dot{f}\|_{L_{p}[0,1]}^{p}=\sum_{j=1}^{s} \sum_{i=1}^{\nu_{j}} \int_{\Delta_{i, j}^{0}}\left|c_{j, i}\right|^{p}\left|\dot{\varphi}_{i, j}^{0}(t)\right|^{p} d t \underset{p, q}{\asymp} \sum_{j=1}^{s} \sum_{i=1}^{\nu_{j}}\left|c_{j, i}\right|^{p}\left(s \nu_{j}\right){ }^{\left(1+\frac{1}{q}-\frac{1}{p}\right) p} \stackrel{(1.11)}{\leq} 1, \\
& \|\dot{f}\|_{L_{1}\left[\frac{j-1}{s}, \frac{j}{s}\right]}=\sum_{i=1}^{\nu_{j}}\left|c_{j, i}\right| \int_{\Delta_{i, j}^{0}}\left|\dot{\varphi}_{i, j}^{0}(t)\right| d t \underset{q}{\asymp} \sum_{i=1}^{\nu_{j}}\left|c_{j, i}\right|\left(s \nu_{j}\right)^{1 / q} \stackrel{(1.11)}{\leq} \frac{\varepsilon_{j}}{s}, \quad 1 \leq j \leq s .
\end{aligned}
$$

Следовательно, существует $a(p, q)>0$ такое, что $a(p, q) f \stackrel{(0.2)}{\in} M$. 
В силу (1.2)

$$
\left\|\sum_{j=1}^{s} \sum_{i=1}^{\nu_{j}} c_{j, i} \varphi_{i, j}^{0}\right\|_{L_{q}[0,1]}=\left\|\left(c_{i, j}\right)_{1 \leq j \leq s, 1 \leq i \leq \nu_{j}}\right\|_{l_{q}^{n}}
$$

Рассуждая так же, как в [16], получаем, что $d_{[n / 2]}\left(M, L_{q}[0,1]\right) \underset{p, q}{\gtrsim} d_{[n / 2]}\left(V, l_{q}^{n}\right)$. Поэтому достаточно показать, что

$$
d_{[n / 2]}\left(V, l_{q}^{n}\right) \underset{p, q}{\gtrsim} \begin{cases}n^{-1}\left(\sum_{j=1}^{s} \frac{\varepsilon_{j}^{\frac{1}{\theta+1}}}{s}\right)^{(\theta+1) \frac{p-2}{2(p-1)}}, & p>2, q \geq 2, \\ n^{-1}\left(\sum_{j=1}^{s} \frac{\varepsilon_{j}^{\frac{1}{\theta+1}}}{s}\right)^{(\theta+1) \frac{p-q}{q(p-1)}}, & p>q, q \leq 2, \\ n^{-\max \{1+1 / q-1 / p, 1+1 / 2-1 / p\}}, & p \leq q, p \leq 2,\end{cases}
$$

где $\theta$ задано формулой (1.10). Это будет сделано в разд. 4.

Теперь рассмотрим класс $\tilde{M}$.

Дискретизачия для оценки сверху. Операторы $P_{m}$ определяем так же, как и раньше. Аналогично соотношению (1.4) доказывается, что для $f \in \tilde{M}$

$$
\max _{1 \leq j \leq s} 2^{m} \nu_{j} s\left\|P_{m+1} f-P_{m} f\right\|_{L_{\infty}\left(\Delta_{j, i}^{m}\right)} \lesssim 1 .
$$

Формула (1.5) в этом случае тоже верна, откуда получаем аналог (1.6)

$$
\max _{1 \leq j \leq s}\left(2^{m} \nu_{j} s\right)^{1+\frac{1}{q}} \max _{1 \leq i \leq 2^{m} \nu_{j}}\left|c_{j, i}^{m}\right| \underset{q}{\lesssim} 1
$$

Напомним, что $\varepsilon_{j}$ заданы формулой (0.3).

Покажем, что

$$
\sum_{i=1}^{2^{m} \nu_{j}}\left|c_{j, i}^{m}\right| \underset{q}{\lesssim} \frac{\varepsilon_{j}}{s} \frac{1}{\left(2^{m} \nu_{j} s\right)^{1 / q}}
$$

Пусть $\left|f\left(t_{i, j}^{m}\right)-f\left(t_{i-1, j}^{m}\right)\right|=\frac{1-\alpha_{i, j}^{m}}{2^{m} \nu_{j} s}, \alpha_{i, j}^{m} \in[0,1]$. Тогда

$$
\left|c_{j, i}^{m}\right| \cdot\left|\varphi^{m}\left(\tau_{i, j}^{m}\right)\right|=\left|P_{m+1} f\left(\tau_{i, j}^{m}\right)-P_{m} f\left(\tau_{i, j}^{m}\right)\right|=\left|f\left(\tau_{i, j}^{m}\right)-\frac{f\left(t_{i, j}^{m}\right)+f\left(t_{i-1, j}^{m}\right)}{2}\right| \lesssim \frac{\alpha_{i, j}^{m}}{2^{m} \nu_{j} s} .
$$

Значит,

$$
\sum_{i=1}^{2^{m} \nu_{j}}\left|c_{j, i}^{m}\right| \cdot\left|\varphi_{i, j}^{m}\left(\tau_{i, j}^{m}\right)\right| \underset{q}{\lesssim} \sum_{i=1}^{2^{m} \nu_{j}} \frac{\alpha_{i, j}^{m}}{2^{m} \nu_{j} s}
$$

Далее,

$$
\frac{1}{s}\left(1-\varepsilon_{j}\right) \stackrel{(0.3)}{=}\left|y_{j}-y_{j-1}\right| \leq \sum_{i=1}^{2^{m} \nu_{j}}\left|f\left(t_{i, j}^{m}\right)-f\left(t_{i-1, j}^{m}\right)\right|=\sum_{i=1}^{2^{m} \nu_{j}}\left(2^{m} \nu_{j} s\right)^{-1}\left(1-\alpha_{i, j}^{m}\right)=\frac{1}{s}-\left(2^{m} \nu_{j} s\right)^{-1} \sum_{i=1}^{2^{m} \nu_{j}} \alpha_{i, j}^{m},
$$

поэтому $\sum_{i=1}^{2^{m} \nu_{j}}\left(2^{m} \nu_{j} s\right)^{-1} \alpha_{i, j}^{m} \leq \frac{\varepsilon_{j}}{s}$. Отсюда и из (1.1), (1.15) получаем (1.14).

Пусть $V_{m}-$ множество последовательностей $\left(c_{j, i}^{m}\right)_{1 \leq j \leq s, 1 \leq i \leq 2^{m} \nu_{j}}$ таких, что

$$
\max _{1 \leq j \leq s}\left(2^{m} \nu_{j} s\right)^{1+\frac{1}{q}} \max _{1 \leq i \leq 2^{m} \nu_{j}}\left|c_{j, i}^{m}\right| \leq 1
$$




$$
\sum_{i=1}^{2^{m} \nu_{j}}\left|c_{j, i}^{m}\right| \underset{q}{\lesssim} \frac{\varepsilon_{j}}{s} \frac{1}{\left(2^{m} \nu_{j} s\right)^{1 / q}}, \quad 1 \leq j \leq s
$$

Рассуждая, как в [16], получаем, что достаточно показать, что найдутся числа $\nu_{j} \in \mathbb{N}, k(m) \in \mathbb{Z}_{+}$ такие, что $\sum_{j=1}^{s} \nu_{j}=n, \sum_{m \in \mathbb{Z}_{+}} k(m)=n$,

$$
\sum_{m \in \mathbb{Z}_{+}} d_{k(m)}\left(V_{m}, l_{q}^{2^{m} n}\right) \underset{q}{\lesssim} \begin{cases}n^{-1}\left(\sum_{j=1}^{s} \frac{\varepsilon_{j}^{\frac{1}{\theta+1}}}{s}\right)^{\frac{\theta+1}{2}}, & q \geq 2, \\ n^{-1}\left(\sum_{j=1}^{s} \frac{\varepsilon_{j}^{\frac{1}{\theta+1}}}{s}\right)^{\frac{\theta+1}{q}}, \quad q<2,\end{cases}
$$

где

$$
\theta= \begin{cases}\frac{q+2}{q}, & q \geq 2 \\ q, & q<2 .\end{cases}
$$

Эта оценка будет получена в разд. 3.

Дискретизация для оценки снизу. Пусть $\Lambda$ - пространство непрерывных кусочно-линейных функций с узлами в $t_{i, j}^{0}, 1 \leq j \leq s, 1 \leq i \leq \nu_{j}$. Тогда $\operatorname{dim} \Lambda=n+1$ и

$$
d_{2 n+1}\left(\tilde{M}+\Lambda, L_{q}[0,1]\right) \leq d_{n}\left(\tilde{M}, L_{q}[0,1]\right) .
$$

Оценим снизу левую часть этого неравенства.

Положим $k_{j}=\left[\varepsilon_{j} \nu_{j}\right], 1 \leq j \leq s, I_{j} \subset\left\{1, \ldots, \nu_{j}\right\}$ - подмножество мощности $k_{j}$,

$$
\mathcal{N}_{i, j}=\left\{\lambda \in \overline{1,4 \nu_{j}}: \Delta_{\lambda, j}^{2} \subset \Delta_{i, j}^{0}\right\} .
$$

Покажем, что существует $a(q)>0$ такое, что для любых $z_{\lambda, i, j} \in\{1,-1\}, \lambda \in \mathcal{N}_{i, j}$, выполнено

$$
\varphi=a(q) \sum_{j=1}^{s} \sum_{i \in I_{j}} \sum_{\lambda \in \mathcal{N}_{i, j}} \frac{z_{\lambda, i, j}}{\left(4 s \nu_{j}\right)^{1+\frac{1}{q}}} \varphi_{\lambda, j}^{2} \in \tilde{M}+\Lambda
$$

В самом деле, $\left|\frac{\dot{\varphi}_{\lambda, j}^{2}(t)}{\left(4 s \nu_{j}\right)^{1+\frac{1}{q}}}\right| \underset{q}{\stackrel{(1.1)}{\lesssim}} 1$ п.в., поэтому найдется такое $a(q)>0$, что $|\dot{\varphi}(t)| \leq 1$ п.в. Пусть $f$ - кусочно-линейная непрерывная функция, $f(0)=y_{0},\left.\dot{f}\right|_{\left[t_{i-1, j}^{0}, t_{i, j}^{0}\right]}=0, i \in I_{j}$,

$$
\left.\dot{f}\right|_{\left[t_{i-1, j}^{0}, t_{i, j}^{0}\right]}=\frac{y_{j}-y_{j-1}}{\nu_{j}-k_{j}} \nu_{j} s, \quad i \notin I_{j} .
$$

Тогда $f \in \Lambda$. Далее, $|\dot{f}(t)| \stackrel{(0.3)}{\leq} 1$ и $f\left(t_{j}\right)=y_{j}, 1 \leq j \leq s$. Наконец, носители у $\dot{\varphi}$ и $\dot{f}$ не перекрываются. Значит, $f+\varphi \in \tilde{M}$, откуда $\varphi=(f+\varphi)-f \in \tilde{M}+\Lambda$.

$\mathrm{B} \mathbb{R}^{4 n}$ координаты занумеруем двойными индексами $(j, \lambda)$, где $1 \leq j \leq s, 1 \leq \lambda \leq 4 \nu_{j}$. Пусть $e_{j, \lambda}$ - вектор, у которого на месте $(j, \lambda)$ стоит 1 , а на остальных местах нули,

$$
V=\operatorname{conv}\left\{\sum_{j=1}^{s} \sum_{i \in I_{j}} \sum_{\lambda \in \mathcal{N}_{i, j}} \frac{z_{\lambda, i, j}}{\left(4 s \nu_{j}\right)^{1+\frac{1}{q}}} e_{j, \lambda}: z_{\lambda, i, j}= \pm 1, \quad I_{j} \subset \overline{1, \nu_{j}}, \quad\left|I_{j}\right|=k_{j}\right\},
$$

где conv обозначает выпуклую оболочку. Достаточно показать, что существуют $\nu_{j} \in \mathbb{N}$ такие, что $\sum_{j=1}^{s} \nu_{j}=n$,

$$
d_{2 n+1}\left(V, l_{q}^{4 n}\right) \underset{q}{\gtrsim} \begin{cases}n^{-1}\left(\sum_{j=1}^{s} \frac{\varepsilon_{j}^{\frac{1}{\theta+1}}}{s}\right)^{\frac{\theta+1}{2}}, & q \geq 2, \\ n^{-1}\left(\sum_{j=1}^{s} \frac{\varepsilon_{j}^{\frac{1}{\theta+1}}}{s}\right)^{\frac{\theta+1}{q}}, & q<2,\end{cases}
$$

где $\theta$ задано формулой (1.16). 


\section{2. Оценки сверху поперечников конечномерных множеств}

Без ограничения общности можно считать, что $\varepsilon_{j}>0,1 \leq j \leq s$.

Через $B_{p}^{\nu}$ обозначим единичный шар в пространстве $l_{p}^{\nu}$.

Для чисел $\alpha \in \mathbb{R}$ и $1 \leq p \leq \infty$ положим $B l^{\nu}(1 / p, \alpha)=\left\{x \in \mathbb{R}^{\nu}:\|x\|_{l_{p}^{\nu}} \leq \nu^{-\alpha}\right\}$. Пусть $K \subset[0,1] \times \mathbb{R}$ - компакт. Положим $B l^{\nu}(K)=\cap_{(1 / p, \alpha) \in K} B l^{\nu}(1 / p, \alpha), G(K)=$ conv $K+$ cone $\{(-1,0),(1,-1)\}$, где сопе обозначает коническую оболочку. Э. М. Галеевым $[5$, теорема 2] был получен следующий результат. Пусть $K_{1}, K_{2} \subset[0,1] \times \mathbb{R}-$ компакты. Тогда $B l^{\nu}\left(K_{1}\right) \subset B l^{\nu}\left(K_{2}\right)$ тогда и только тогда, когда $G\left(K_{2}\right) \subset G\left(K_{1}\right)$.

Из этой теоремы следует, что если $(1 / q, \beta) \in[(1 / p, \gamma),(1, \alpha)]$, то $B l^{\nu}(1 / p, \gamma) \cap B l^{\nu}(1, \alpha) \subset$ $B l^{\nu}(1 / q, \beta)$.

Следствие 1. Пусть $p>1, \nu \in \mathbb{N}, \varepsilon>0, \gamma \in \mathbb{R}$. Положим

$$
E=\left\{x \in \mathbb{R}^{\nu}:\|x\|_{l_{p}^{\nu}} \leq \nu^{-\gamma},\|x\|_{l_{1}^{\nu}} \leq \varepsilon \nu\right\} .
$$

1. Пусть $p>2$. Тогда $E \subset \nu^{\frac{p-2}{2(p-1)}-\gamma \frac{p}{2(p-1)}} \varepsilon^{\frac{p-2}{2(p-1)}} B_{2}^{\nu}$.

2. Пусть $1<q \leq 2, p>q$. Тогда $E \subset \nu^{\frac{p-q}{q(p-1)}-\gamma \frac{p(q-1)}{q(p-1)}} \varepsilon^{\frac{p-q}{q(p-1)}} B_{q}^{\nu}$.

Д о к аз а т е ль с т в о. Пусть $\varepsilon \nu=\nu^{-\alpha}$. В случае $p>2$ выполнено включение $E \subset$ $\nu^{-\beta} B_{2}^{\nu}$, где $\beta$ находится из условий

$$
\frac{\lambda}{p}+1-\lambda=\frac{1}{2}, \quad \lambda \gamma+(1-\lambda) \alpha=\beta .
$$

Тогда $\lambda=\frac{p}{2(p-1)}, 1-\lambda=\frac{p-2}{2(p-1)}$,

$$
\nu^{-\beta}=\nu^{-\lambda \gamma-(1-\lambda) \alpha}=\nu^{1-\lambda-\lambda \gamma} \varepsilon^{1-\lambda}=\nu^{\frac{p-2}{2(p-1)}-\gamma \frac{p}{2(p-1)}} \varepsilon^{\frac{p-2}{2(p-1)}} .
$$

В случае $1<q \leq 2, p>q$ имеем $E \subset \nu^{-\beta} B_{q}^{\nu}$, где $\beta$ находится из условий

$$
\frac{\lambda}{p}+1-\lambda=\frac{1}{q}, \quad \lambda \gamma+(1-\lambda) \alpha=\beta .
$$

Тогда $\lambda=\frac{1-1 / q}{1-1 / p}, 1-\lambda=\frac{1 / q-1 / p}{1-1 / p}$,

$$
\nu^{-\beta}=\nu^{-\frac{1-\frac{1}{q}}{1-\frac{1}{p}} \gamma}(\varepsilon \nu)^{\frac{\frac{1}{q}-\frac{1}{p}}{1-\frac{1}{p}}}=\varepsilon^{\frac{p-q}{q(p-1)}} \nu^{-\frac{p(q-1)}{q(p-1)} \gamma+\frac{p-q}{q(p-1)}} .
$$

Следствие доказано.

Перейдем к задаче оценки поперечников множеств $V_{m}$, определенных в предыдущем разделе.

Случай $p>2, q \geq 2$. Оценим сверху по порядку радиус евклидова шара, содержащего $V_{m}$. Полученную величину минимизируем по $\left(\nu_{j}\right)_{j=1}^{s}$ (задача минимизации окажется одной и той же для всех $m$ ). Считаем, что $\nu_{j} \geq 2$.

Сначала рассмотрим задачу для множества $M$.

Перепишем условие (1.8) в виде

$$
\begin{aligned}
& \sum_{\substack{j=1 \\
2^{m} \nu_{j}}}^{s}\left(2^{m} \nu_{j} s\right)^{\left(1+\frac{1}{q}\right) p}\left(2^{m} \nu_{j} s\right)^{-1} \sum_{i=1}^{2^{m} \nu_{j}}\left|c_{j, i}^{m}\right|^{p} \leq 1, \\
& \sum_{i=1}^{m}\left|c_{j, i}^{m}\right| \leq \frac{\varepsilon_{j}}{\left(2^{m} \nu_{j} s\right)^{1+\frac{1}{q}}} 2^{m} \nu_{j}, \quad 1 \leq j \leq s .
\end{aligned}
$$


Фиксируем $1 \leq j \leq s$. Пусть $\gamma_{j} \in \mathbb{R}$ таково, что

$$
\begin{gathered}
\sum_{i=1}^{2^{m} \nu_{j}}\left|c_{j, i}^{m}\right|^{p}=\left(2^{m} \nu_{j}\right)^{-\gamma_{j} p}\left(2^{m} \nu_{j} s\right)^{-\left(1+\frac{1}{q}\right) p}, \\
\sum_{i=1}^{2^{m} \nu_{j}}\left|c_{j, i}^{m}\right| \leq \varepsilon_{j} 2^{m} \nu_{j}\left(2^{m} \nu_{j} s\right)^{-\left(1+\frac{1}{q}\right)} .
\end{gathered}
$$

Применяя следствие 1 , получаем

$$
\sum_{i=1}^{2^{m} \nu_{j}}\left|c_{j, i}^{m}\right|^{2} \leq \frac{1}{\left(2^{m} \nu_{j} s\right)^{2\left(1+\frac{1}{q}\right)}}\left(2^{m} \nu_{j}\right)^{\frac{1}{p-1}\left(p-2-\gamma_{j} p\right)} \varepsilon_{j}^{\frac{p-2}{p-1}}=: E_{j} .
$$

Подставим первое равенство (2.2) в (2.1) и получим

$$
\sum_{j=1}^{s}\left(2^{m} \nu_{j}\right)^{-1-\gamma_{j} p} \leq s
$$

Чтобы найти минимальный радиус евклидова шара, содержащего $V_{m}$, максимизируем по $\left(\gamma_{1}, \ldots, \gamma_{s}\right)$ величину

$$
L=L\left(\gamma_{1}, \ldots, \gamma_{s}\right)=\sum_{j=1}^{s} \frac{1}{\left(2^{m} \nu_{j} s\right)^{2\left(1+\frac{1}{q}\right)}}\left(2^{m} \nu_{j}\right)^{\frac{1}{p-1}\left(p-2-\gamma_{j} p\right)} \varepsilon_{j}^{\frac{p-2}{p-1}}
$$

при условии, что выполнено (2.4).

Положив $\sigma_{j}=\left(2^{m} \nu_{j}\right)^{-1-\gamma_{j} p}$, получаем

$$
L=\sum_{j=1}^{s}\left(2^{m} \nu_{j} s\right)^{-2\left(1+\frac{1}{q}\right)+1} \sigma_{j}^{\frac{1}{p-1}} \varepsilon_{j}^{\frac{p-2}{p-1}} \cdot \frac{1}{s}=\sum_{j=1}^{s}\left(2^{m} \nu_{j} s\right)^{-1-\frac{2}{q}} \varepsilon_{j}^{\frac{p-2}{p-1}} \sigma_{j}^{\frac{1}{p-1}} \cdot \frac{1}{s} .
$$

Пусть $\tau_{j}=\sigma_{j}^{\frac{1}{p-1}}, a_{j}=\left(2^{m} \nu_{j} s\right)^{-1-\frac{2}{q}} \varepsilon_{j}^{\frac{p-2}{p-1}} \frac{1}{s}$. Тогда задача минимизации $L\left(\gamma_{1}, \ldots, \gamma_{s}\right)$ при условии (2.4) эквивалентна следующей:

$$
\sum_{j=1}^{s} a_{j} \tau_{j} \rightarrow \max , \quad \sum_{j=1}^{s} \tau_{j}^{p-1} \leq s
$$

Так как $p>2$, то в силу неравенства Гёльдера

$$
\sum_{j=1}^{s} a_{j} \tau_{j} \leq\left(\sum_{j=1}^{s} a_{j}^{\frac{p-1}{p-2}}\right)^{\frac{p-2}{p-1}} s^{\frac{1}{p-1}}
$$

Подставив выражения для $a_{j}$, получаем, что для радиуса $R$ описанного евклидова шара выполнено

$$
R^{2} \leq\left(\sum_{j=1}^{s}\left(2^{m} \nu_{j} s\right)^{-\frac{q+2}{q} \cdot \frac{p-1}{p-2}} \varepsilon_{j}\right)^{\frac{p-2}{p-1}} s^{\frac{1}{p-1}-1} .
$$

Отметим также, что в точке максимума выполнено

$$
\tau_{j}=s^{\frac{1}{p-1}} \frac{a_{j}^{\frac{1}{p-2}}}{\left(\sum_{k=1}^{s} a_{k}^{\frac{p-1}{p-2}}\right)^{\frac{1}{p-1}}}, \quad \tau_{j}=\left(2^{m} \nu_{j}\right)^{-\frac{1+\gamma_{j} p}{p-1}}, \quad a_{j}=\left(2^{m} \nu_{j} s\right)^{-1-\frac{2}{q}} \varepsilon_{j}^{\frac{p-2}{p-1}} \frac{1}{s}
$$


откуда

$$
E_{j}=a_{j} \tau_{j}=s^{\frac{1}{p-1}} \frac{a_{j}^{\frac{p-1}{p-2}}}{\left(\sum_{k=1}^{s} a_{k}^{\frac{p-1}{p-2}}\right)^{\frac{1}{p-1}}}=s^{\frac{1}{p-1}-1-\frac{q+2}{q}} \frac{\left(2^{m} \nu_{j}\right)^{-\frac{q+2}{q} \cdot \frac{p-1}{p-2}} \varepsilon_{j}}{\left(\sum_{k=1}^{s}\left(2^{m} \nu_{k}\right)^{-\frac{q+2}{q} \cdot \frac{p-1}{p-2}} \varepsilon_{k}\right)^{\frac{1}{p-1}}} .
$$

Итак, при фиксированных $\nu_{1}, \ldots, \nu_{s} \in \mathbb{N}$ таких, что $\sum_{j=1}^{s} \nu_{j}=n$, множество $V_{m}$ содержится в евклидовом шаре радиуса $R$, удовлетворяющего (2.6). Минимизируем правую часть (2.6) по $\nu_{j}$ (отметим, что точка минимума будет одна и та же при всех $m \in \mathbb{Z}_{+}$). Получаем задачу

$$
\sum_{j=1}^{s}\left(\nu_{j} s\right)^{-\frac{q+2}{q} \cdot \frac{p-1}{p-2}} \varepsilon_{j} \rightarrow \min , \quad \sum_{j=1}^{s} \nu_{j}=n, \quad \nu_{j} \in \mathbb{N}, \quad \nu_{j} \geq 2 .
$$

Положим

$$
\theta=\frac{q+2}{q} \frac{p-1}{p-2}
$$

Тогда (2.9) переписывается в виде

$$
\sum_{j=1}^{s} \varepsilon_{j} \nu_{j}^{-\theta} \rightarrow \min , \quad \sum_{j=1}^{s} \nu_{j}=n, \quad \nu_{j} \in \mathbb{N}, \quad \nu_{j} \geq 2 .
$$

Сначала найдем точку минимума на множестве $\nu_{j}>0(1 \leq j \leq s), \sum_{j=1}^{s} \nu_{j}=n$. Применяя принцип Лагранжа и учитывая, что минимизируется выпуклая функция на выпуклом множестве, получаем, что минимум достигается в точке $\left(\hat{\nu}_{1}, \ldots, \hat{\nu}_{s}\right)$, для которой выполняется $\varepsilon_{j} \hat{\nu}_{j}^{-\theta-1}=$ const. Значит,

$$
\hat{\nu}_{j}=n \frac{\varepsilon_{j}^{\frac{1}{\theta+1}}}{\sum_{i=1}^{s} \varepsilon_{i}^{\frac{1}{\theta+1}}} .
$$

Если $n$ достаточно велико, то выбираем $\nu_{j} \in \mathbb{N}$ такие, что $\sum_{j=1}^{s} \nu_{j}=n, \nu_{j} \geq 2$,

$$
\frac{\hat{\nu}_{j}}{2} \leq \nu_{j} \leq 2 \hat{\nu}_{j}
$$

и получаем

$$
\sum_{j=1}^{s}\left(2^{m} s\right)^{-\theta} \nu_{j}^{-\theta} \varepsilon_{j} \underset{p, q}{\asymp}\left(2^{m} n s\right)^{-\theta}\left(\sum_{j=1}^{s} \varepsilon_{j}^{\frac{1}{\theta+1}}\right)^{\theta+1}
$$

Таким образом, при больших $n$ оценка для радиуса описанной сферы имеет вид

$$
R \underset{p, q}{\lesssim}\left(2^{m} n\right)^{-\frac{q+2}{2 q}}\left(\sum_{j=1}^{s} \frac{\varepsilon_{j}^{\frac{1}{\theta+1}}}{s}\right)^{(\theta+1) \frac{p-2}{2(p-1)}}
$$

Перейдем к доказательству неравенства (1.9). При выбранных $\nu_{j}$ имеем

$$
\sum_{m \in \mathbb{Z}_{+}} d_{k(m)}\left(V_{m}, l_{q}^{N_{m}}\right) \underset{p, q}{\lesssim}\left(\sum_{j=1}^{s} \frac{\varepsilon_{j}^{\frac{1}{\theta+1}}}{s}\right)^{(\theta+1) \frac{p-2}{2(p-1)}} \sum_{m \in \mathbb{Z}_{+}}\left(2^{m} n\right)^{-\frac{q+2}{2 q}} d_{k(m)}\left(B_{2}^{2^{m} n}, l_{q}^{2^{m} n}\right) .
$$

Числа $k(m) \in \mathbb{Z}_{+}$подбираем так, чтобы $\sum_{m \in \mathbb{Z}_{+}} k(m) \lesssim n$. Напомним, что $q \geq 2$. Рассуждая так же, как при оценке поперечников классов Соболева [16], получаем, что при достаточно больших $n$ выполнено

$$
\sum_{m \in \mathbb{Z}_{+}} d_{k(m)}\left(V_{m}, l_{q}^{N_{m}}\right) \underset{p, q}{\lesssim} n^{-1}\left(\sum_{j=1}^{s} \frac{\varepsilon_{j}^{\frac{1}{\theta+1}}}{s}\right)^{(\theta+1) \frac{p-2}{2(p-1)}} .
$$


Случай $p=\infty, q \geq 2$. Снова оцениваем по порядку радиус евклидова шара, содержащего $V_{m}$. Вместо условия (2.1) имеем

$$
\begin{gathered}
\max _{1 \leq j \leq s}\left(2^{m} \nu_{j} s\right)^{\left(1+\frac{1}{q}\right)} \max _{1 \leq i \leq 2^{m} \nu_{j}}\left|c_{j, i}^{m}\right| \leq 1, \\
\sum_{i=1}^{2^{m} \nu_{j}}\left|c_{j, i}^{m}\right| \leq \frac{\varepsilon_{j}}{\left(2^{m} \nu_{j} s\right)^{1+\frac{1}{q}}} 2^{m} \nu_{j}, \quad 1 \leq j \leq s ;
\end{gathered}
$$

выражение (2.2) заменяется на

$$
\begin{gathered}
\max _{1 \leq i \leq 2^{m} \nu_{j}}\left|c_{j, i}^{m}\right|=\left(2^{m} \nu_{j}\right)^{-\gamma_{j}}\left(2^{m} \nu_{j} s\right)^{-\left(1+\frac{1}{q}\right)}, \\
\sum_{i=1}^{2^{m} \nu_{j}}\left|c_{j, i}^{m}\right| \leq \varepsilon_{j} 2^{m} \nu_{j}\left(2^{m} \nu_{j} s\right)^{-\left(1+\frac{1}{q}\right)} .
\end{gathered}
$$

Применяя следствие 1 , получаем

$$
\sum_{i=1}^{2^{m} \nu_{j}}\left|c_{j, i}^{m}\right|^{2} \leq \frac{1}{\left(2^{m} \nu_{j} s\right)^{2\left(1+\frac{1}{q}\right)}}\left(2^{m} \nu_{j}\right)^{1-\gamma_{j}} \varepsilon_{j} .
$$

Рассуждаем так же, как в предыдущем случае. Для того чтобы найти радиус евклидова шара, содержащего $V_{m}$, максимизируем функцию

$$
L\left(\gamma_{1}, \ldots, \gamma_{s}\right)=\sum_{j=1}^{s} \frac{1}{\left(2^{m} \nu_{j} s\right)^{2\left(1+\frac{1}{q}\right)}}\left(2^{m} \nu_{j}\right)^{1-\gamma_{j}} \varepsilon_{j}
$$

при условии, что $\max _{1 \leq j \leq s}\left(2^{m} \nu_{j}\right)^{-\gamma_{j}} \leq 1$. Полученное выражение минимизируем по $\nu_{j} \in \mathbb{N}$, удовлетворяющим условию $\sum_{j=1}^{s} \nu_{j}=n$. В итоге получаем

$$
\sum_{m \in \mathbb{Z}_{+}} d_{k(m)}\left(V_{m}, l_{q}^{N_{m}}\right) \underset{q}{\lesssim} n^{-1}\left(\sum_{j=1}^{s} \frac{\varepsilon_{j}^{\frac{1}{\theta+1}}}{s}\right)^{\frac{\theta+1}{2}},
$$

где $\theta=\frac{q+2}{q}$.

Случай $p>q, q \leq 2$. Найдем радиус шара в $l_{q}^{2^{m} n}$, содержащего множество последовательностей $\left(c_{j, i}\right)_{1 \leq j \leq s, 1 \leq i \leq 2^{m} \nu_{j}}$, удовлетворяющих (2.1), и минимизируем его за счет выбора $\nu_{j}$ (снова точка минимума не будет зависеть от $m$ ).

Для фиксированного $j$ рассмотрим множество последовательностей, удовлетворяющих условиям (2.2). Применяя следствие 1 , получаем

$$
\sum_{i=1}^{2^{m} \nu_{j}}\left|c_{j, i}^{m}\right|^{q} \leq \varepsilon_{j}^{\frac{p-q}{p-1}}\left(2^{m} \nu_{j}\right)^{-\frac{p(q-1)}{p-1} \gamma_{j}+\frac{p-q}{p-1}-q-1} s^{-q-1}=: E_{j} .
$$

Для нахождения радиуса описанного $l_{q}$-шара решаем задачу на максимум

$$
\sum_{j=1}^{s} s^{-q-1} \varepsilon_{j}^{\frac{p-q}{p-1}}\left(2^{m} \nu_{j}\right)^{\frac{p-q}{p-1}-q-1-\frac{p(q-1)}{p-1} \gamma_{j}} \rightarrow \max , \quad \sum_{j=1}^{s}\left(2^{m} \nu_{j}\right)^{-1-\gamma_{j} p} \leq s .
$$

Положим $\sigma_{j}=\left(2^{m} \nu_{j}\right)^{-1-\gamma_{j} p}$. Тогда (2.18) переписывается в виде

$$
\sum_{j=1}^{s} s^{-q-1} \varepsilon_{j}^{\frac{p-q}{p-1}}\left(2^{m} \nu_{j}\right)^{-q} \sigma_{j}^{\frac{q-1}{p-1}} \rightarrow \max , \quad \sum_{j=1}^{s} \sigma_{j} \leq s .
$$


Положим $\tau_{j}=\sigma_{j}^{\frac{q-1}{p-1}}, a_{j}=s^{-q-1} \varepsilon_{j}^{\frac{p-q}{p-1}}\left(2^{m} \nu_{j}\right)^{-q}$. Применив неравенство Гельдера и учитывая неравенство $\sum_{j=1}^{s} \tau_{j}^{\frac{p-1}{q-1}} \leq s$, получим

$$
\sum_{j=1}^{s} a_{j} \tau_{j} \leq s^{\frac{q-1}{p-1}}\left(\sum_{j=1}^{s} a_{j}^{\frac{p-1}{p-q}}\right)^{\frac{p-q}{p-1}}=s^{-q-1+\frac{q-1}{p-1}}\left(\sum_{j=1}^{s} \varepsilon_{j}\left(2^{m} \nu_{j}\right)^{-\frac{q(p-1)}{p-q}}\right)^{\frac{p-q}{p-1}} .
$$

Отметим, что в точке максимума выполнено

$$
\tau_{j}=\frac{a_{j}^{\frac{q-1}{p-q}}}{\left(\sum_{k=1}^{s} a_{k}^{\frac{p-1}{p-q}}\right)^{\frac{q-1}{p-1}}} s^{\frac{q-1}{p-1}}, \quad \tau_{j}=\left(2^{m} \nu_{j}\right)^{-\frac{\left(1+\gamma_{j} p\right)(q-1)}{p-1}}, \quad a_{j}=s^{-q-1} \varepsilon_{j}^{\frac{p-q}{p-1}}\left(2^{m} \nu_{j}\right)^{-q},
$$

откуда

$$
E_{j}=a_{j} \tau_{j}=\frac{a_{j}^{\frac{p-1}{p-q}}}{\left(\sum_{k=1}^{s} a_{k}^{\frac{p-1}{p-q}}\right)^{\frac{q-1}{p-1}}} s^{\frac{q-1}{p-1}}=\frac{\left(2^{m} \nu_{j}\right)^{-\frac{q(p-1)}{p-q}} \varepsilon_{j}}{\left(\sum_{k=1}^{s}\left(2^{m} \nu_{k}\right)^{-\frac{q(p-1)}{p-q}} \varepsilon_{k}\right)^{\frac{q-1}{p-1}}} s^{\frac{q-1}{p-1}-q-1} .
$$

Теперь минимизируем радиус описанного шара по $\nu_{j} \in \mathbb{N}, \sum_{j=1}^{s} \nu_{j}=n$ (заметим, что точка минимума не зависит от $\left.m \in \mathbb{Z}_{+}\right)$. Получаем задачу

$$
\sum_{j=1}^{s} \varepsilon_{j} \nu_{j}^{-\frac{q(p-1)}{p-q}} \rightarrow \min , \quad \sum_{j=1}^{s} \nu_{j}=n, \quad \nu_{j} \in \mathbb{N}, \quad \nu_{j} \geq 2 .
$$

Положим $\theta=\frac{q(p-1)}{p-q}$. Сначала рассмотрим ту же задачу, заменив условия $\nu_{j} \in \mathbb{N}, \nu_{j} \geq 2$, на неравенства $\nu_{j}>0$. Применяя принцип Лагранжа и учитывая, что минимизируется выпуклая функция на выпуклом множестве, получаем, что минимум достигается в точке $\left(\hat{\nu}_{1}, \ldots, \hat{\nu}_{s}\right)$, где

$$
\hat{\nu}_{j}=n \frac{\varepsilon_{j}^{\frac{1}{\theta+1}}}{\sum_{i=1}^{s} \varepsilon_{i}^{\frac{1}{\theta+1}}} .
$$

Если $n \in \mathbb{N}$ достаточно велико, то найдутся $\nu_{j} \in \mathbb{N}$ такие, что $\sum_{j=1}^{s} \nu_{j}=n$ и $\hat{\nu}_{j} / 2 \leq \nu_{j} \leq 2 \hat{\nu}_{j}$, при этом $\nu_{j} \geq 2$. Тогда

$$
\sum_{j=1}^{s} \varepsilon_{j}\left(2^{m} \nu_{j}\right)^{-\theta} \underset{\overline{p, q}}{\asymp} 2^{-m \theta} n^{-\theta}\left(\sum_{j=1}^{s} \varepsilon_{j}^{\frac{1}{\theta+1}}\right)^{\theta+1}
$$

В итоге получаем, что если $\left(c_{j, i}^{m}\right)_{1 \leq j \leq s, 1 \leq i \leq \nu_{j}} \in V_{m}$, то

$$
\left(\sum_{j=1}^{s} \sum_{i=1}^{2^{m} \nu_{j}}\left|c_{j, i}^{m}\right|^{q}\right)^{1 / q} \lesssim\left(2^{m} n\right)^{-1}\left(\frac{1}{s} \sum_{j=1}^{s} \varepsilon_{j}^{\frac{1}{\theta+1}}\right)^{(\theta+1) \frac{p-q}{q(p-1)}} .
$$

Положим $k(m)=0$ при $m \geq 1, k(0)=n$. Тогда

$$
\sum_{m \in \mathbb{Z}_{+}} d_{k(m)}\left(V_{m}, l_{q}^{N_{m}}\right) \underset{p, q}{\underset{m}{\lesssim}} \sum_{m \in \mathbb{N}}\left(2^{m} n\right)^{-1}\left(\frac{1}{s} \sum_{j=1}^{s} \varepsilon_{j}^{\frac{1}{\theta+1}}\right)^{(\theta+1) \frac{p-q}{q(p-1)}} \underset{p, q}{\underset{j}{\lesssim}} n^{-1}\left(\frac{1}{s} \sum_{j=1}^{s} \varepsilon_{j}^{\frac{1}{\theta+1}}\right)^{(\theta+1) \frac{p-q}{q(p-1)}} .
$$

Случай $p=\infty, q \leq 2$. Найдем радиус шара в $l_{q}^{N_{m}}$, содержащего множество последовательностей $\left(c_{j, i}^{m}\right)_{1 \leq j \leq s, 1 \leq i \leq \nu_{j}}$, удовлетворяющих $(2.14)$, и минимизируем его за счет выбора $\nu_{j}$. Для 
фиксированного $j$ рассматриваем множество последовательностей, удовлетворяющих (2.15). Применяя следствие 1 , получаем

$$
\sum_{i=1}^{2^{m} \nu_{j}}\left|c_{j, i}^{m}\right|^{q} \leq \varepsilon_{j}\left(2^{m} \nu_{j}\right)^{-(q-1) \gamma_{j}-q} s^{-q-1} .
$$

Рассуждая так же, как в предыдущих случаях, имеем

$$
\sum_{m \in \mathbb{Z}_{+}} d_{k(m)}\left(V_{m}, l_{q}^{N_{m}}\right) \underset{q}{\lesssim} n^{-1}\left(\sum_{j=1}^{s} \frac{\varepsilon_{j}^{\frac{1}{\theta+1}}}{s}\right)^{\frac{\theta+1}{q}}
$$

где $\theta=q$.

3 а м е ч а н и е. Во всех случаях $\gamma_{j}$, максимизирующее длину вектора, строго больше -1 при $m=0$ и достаточно больших $n$. В самом деле, в силу (2.7), (2.12), (2.20), (2.23) получаем, что $\tau_{j}=n^{-\frac{\left(1+\gamma_{j} p\right)(\min \{q, 2\}-1)}{p-1}}$ и $\tau_{j} \asymp 1$. Значит, $\gamma_{j}=\gamma_{j}(n) \rightarrow-\frac{1}{p}, n \rightarrow \infty$.

\section{3. Оценка снизу поперечников конечномерных множеств}

Случаи $p>2, q>2$ и $p>q, q \leq 2$. Доказательство является обобщением рассуждений из работы Е. Д. Глускина [9].

Лемма 1. Пусть $\varepsilon>0, \nu \in \mathbb{N}$ таковы, что $\varepsilon \nu^{1+\gamma} \geq 1,1<p \leq \infty$.

1. Пусть $p>2$. Тогда существуют $k \in \mathbb{N}$ и с $>0$ такие, что для вектора $\left(c_{i}\right)_{i=1}^{\nu}$,

$$
c_{i}= \begin{cases}c, & 1 \leq i \leq k \\ 0, & k+1 \leq i \leq \nu\end{cases}
$$

выполнено

$$
\left\|\left(c_{i}\right)_{i=1}^{\nu}\right\|_{l_{p}^{\nu}} \leq \nu^{-\gamma}, \quad\left\|\left(c_{i}\right)_{i=1}^{\nu}\right\|_{l_{1}^{\nu}} \leq \varepsilon \nu, \quad\left\|\left(c_{i}\right)_{i=1}^{\nu}\right\|_{l_{2}^{\nu}} \asymp \nu^{-\gamma \frac{p}{2(p-1)}+\frac{p-2}{2(p-1)}} \varepsilon^{\frac{p-2}{2(p-1)}} .
$$

2. Пусть $p>q, q \leq 2$. Тогда существуют $k \in \mathbb{N} u c>0$ такие, что для вектора $\left(c_{i}\right)_{i=1}^{\nu}$,

$$
c_{i}= \begin{cases}c, & 1 \leq i \leq k \\ 0, & k+1 \leq i \leq \nu\end{cases}
$$

выполнено

$$
\left\|\left(c_{i}\right)_{i=1}^{\nu}\right\|_{l_{p}^{\nu}} \leq \nu^{-\gamma}, \quad\left\|\left(c_{i}\right)_{i=1}^{\nu}\right\|_{l_{1}^{\nu}} \leq \varepsilon \nu, \quad\left\|\left(c_{i}\right)_{i=1}^{\nu}\right\|_{l_{q}^{\nu}} \asymp \nu^{-\gamma \frac{p(q-1)}{q(p-1)}+\frac{p-q}{q(p-1)}} \varepsilon^{\frac{p-q}{q(p-1)}} .
$$

Д о к а з а т е ль с т в о. Выберем числа $\hat{k}>0$ и $c>0$ из соотношений $\hat{k}^{1 / p} c=\nu^{-\gamma}$, $\hat{k} c=\varepsilon \nu$. Тогда $\hat{k}^{1-\frac{1}{p}}=\varepsilon \nu^{1+\gamma} \geq 1, \hat{k}^{1 / 2} c=\nu^{-\gamma \frac{p}{2(p-1)}+\frac{p-2}{2(p-1)}} \varepsilon^{\frac{p-2}{2(p-1)}}, \hat{k}^{1 / q} c=\nu^{-\gamma \frac{p(q-1)}{q(p-1)}+\frac{p-q}{q(p-1)}} \varepsilon^{\frac{p-q}{q(p-1)}}$. Число $k=[\hat{k}]$ будет искомым.

Выберем числа $\nu_{j}$ и $\gamma_{j}$ в соответствии с $(2.7),(2.12),(2.13)$ в случае $p>2, q>2$ и в соответствии с $(2.20),(2.23),(2.13)-$ в случае $p>q, q \leq 2$. Положим

$$
\hat{c}_{j, i}= \begin{cases}\hat{c}_{j}, & 1 \leq i \leq k_{j} \\ 0, & k_{j}+1 \leq i \leq \nu_{j} .\end{cases}
$$

Числа $k_{j}$ и $\hat{c}_{j}$ определим в соответствии с леммой 1 так, чтобы

$$
\left\|\left(\hat{c}_{j, i}\right)_{i=1}^{\nu_{j}}\right\|_{l_{p}^{\nu_{j}}} \leq\left(\nu_{j}\right)^{-\gamma_{j}}\left(\nu_{j} s\right)^{-1-\frac{1}{q}}, \quad\left\|\left(\hat{c}_{j, i}\right)_{i=1}^{\nu_{j}}\right\|_{l_{1}^{\nu_{j}}} \leq \nu_{j} \varepsilon_{j}\left(\nu_{j} s\right)^{-1-\frac{1}{q}},
$$


при этом в случае $p>2, q>2$

$$
\left\|\left(\hat{c}_{j, i}\right)_{i=1}^{\nu_{j}}\right\|_{l_{2}^{\nu_{j}}} \asymp \nu_{j}^{-\gamma_{j} \frac{p}{2(p-1)}+\frac{p-2}{2(p-1)}} \varepsilon_{j}^{\frac{p-2}{2(p-1)}}\left(\nu_{j} s\right)^{-1-\frac{1}{q}},
$$

а при $p>q, q \leq 2$

$$
\left\|\left(\hat{c}_{j, i}\right)_{i=1}^{\nu_{j}}\right\|_{l_{q}^{\nu_{j}}} \asymp \nu_{j}^{-\gamma_{j} \frac{p(q-1)}{q(p-1)}+\frac{p-q}{q(p-1)}} \varepsilon_{j}^{\frac{p-q}{q(p-1)}}\left(\nu_{j} s\right)^{-1-\frac{1}{q}} .
$$

Это можно сделать при достаточно больших $n$, поскольку $\gamma_{j}>-1$ при больших $n$ (см. замечание в конце предыдущего раздела) и $\nu_{j} \rightarrow \infty$ при $n \rightarrow \infty$ для фиксированных $\varepsilon_{j}>0$ в силу (2.12), (2.23) и неравенства $\nu_{j} \geq \hat{\nu}_{j} / 2$.

Из определения $E_{j}$ (см. (2.3) и (2.17)), (2.8), (2.12), (2.21), (2.23) получаем, что при $p>2$, $q \geq 2$

$$
\sum_{i=1}^{\nu_{j}} \hat{c}_{j, i}^{2} \underset{p, q}{\asymp} s^{\frac{1}{p-1}-\frac{q+2}{q}-1} \frac{\nu_{j}^{-\theta} \varepsilon_{j}}{\left(\sum_{k=1}^{s} \nu_{k}^{-\theta} \varepsilon_{k}\right)^{\frac{1}{p-1}}} \underset{p, q}{\asymp} n^{-\frac{q+2}{q}}\left(\frac{1}{s} \sum_{k=1}^{s} \varepsilon_{k}^{\frac{1}{\theta+1}}\right)^{(\theta+1) \frac{p-2}{p-1}} \frac{\nu_{j}^{-\theta} \varepsilon_{j}}{\sum_{k=1}^{s} \nu_{k}^{-\theta} \varepsilon_{k}},
$$

а при $p>q, q \leq 2$

$$
\sum_{i=1}^{\nu_{j}}\left|\hat{c}_{j, i}\right|^{q} \underset{p, q}{\asymp} s^{\frac{q-1}{p-1}-q-1} \frac{\nu_{j}^{-\theta} \varepsilon_{j}}{\left(\sum_{k=1}^{s} \varepsilon_{k} \nu_{k}^{-\theta}\right)^{\frac{q-1}{p-1}}} \widetilde{p, q} n^{-q}\left(\frac{1}{s} \sum_{k=1}^{s} \varepsilon_{k}^{\frac{1}{\theta+1}}\right)^{(\theta+1) \frac{p-q}{p-1}} \frac{\nu_{j}^{-\theta} \varepsilon_{j}}{\sum_{k=1}^{s} \nu_{k}^{-\theta} \varepsilon_{k}} .
$$

В силу формул $(2.12),(2.23)$ и неравенств $\frac{\hat{\nu}_{j}}{2} \leq \nu_{j} \leq 2 \hat{\nu}_{j}$ получаем $\nu_{j}^{-\theta-1} \varepsilon_{j} \underset{p, q}{\asymp}$ const (константа не зависит от $j$ ). Поэтому существуют числа $c_{j, i} \geq 0$ такие, что

$$
\left\{\begin{array}{c}
c_{j, i} \underset{p, q}{\asymp} \hat{c}_{j, i}, \\
\nu_{j}^{-1} \sum_{i=1}^{\nu_{j}} c_{j, i}^{2} \text { не зависит от } j \text { при } q \geq 2, \\
\nu_{j}^{-1} \sum_{i=1}^{\nu_{j}}\left|c_{j, i}\right|^{q} \text { не зависит от } j \text { при } q<2,
\end{array}\right.
$$

и вектор $\hat{x}=\left(c_{j, i}\right)_{1 \leq j \leq s, 1 \leq i \leq \nu_{j}} \in V$. В самом деле, по определению множество $V$ состоит из последовательностей, удовлетворяющих условию (1.11), совпадающему с $(2.1)$ при $m=0$. Второе соотношение (2.1) следует из (3.1), а первое - из (2.7), (2.20) и (3.1).

Положим

$$
A= \begin{cases}n^{-\frac{q+2}{q}}\left(\frac{1}{s} \sum_{k=1}^{s} \varepsilon_{k}^{\frac{1}{\theta+1}}\right)^{(\theta+1) \frac{p-2}{p-1}}, & p>2, q \geq 2, \\ n^{-q}\left(\frac{1}{s} \sum_{k=1}^{s} \varepsilon_{k}^{\frac{1}{\theta+1}}\right)^{(\theta+1) \frac{p-q}{p-1}}, & p>q, q \leq 2 .\end{cases}
$$

Обозначим через $\mathcal{S}_{l}$ группу перестановок из $l$ элементов. Положим $G_{j}=\mathcal{S}_{\nu_{j}} \times\{-1,1\}^{\nu_{j}}$, $G=\prod_{j=1}^{s} G_{j}$. Пусть $g \in G, g=\left(\pi_{j}, \varepsilon_{j, 1}, \ldots, \varepsilon_{j, \nu_{j}}\right)_{1 \leq j \leq s}, \varepsilon_{j, i} \in\{1,-1\}, \pi_{j} \in \mathcal{S}_{\nu_{j}}$. Для $x=$ $\left(x_{j, i}\right)_{1 \leq j \leq s, 1 \leq i \leq \nu_{j}} \in \mathbb{R}^{n}$ полагаем $g x=\left(y_{j, i}\right)_{1 \leq j \leq s, 1 \leq i \leq \nu_{j}} \in \mathbb{R}^{n}, y_{j, i}=\varepsilon_{j, i} x_{j, \pi_{j}(i)}$.

Для любого $g \in G$ выполнено $g \hat{x} \in V$. Обозначим

$$
\tilde{V}=\operatorname{conv}\{g \hat{x}: g \in G\} \subset V
$$

и оценим снизу $d_{l}\left(\tilde{V}, l_{q}^{n}\right), l=[n / 2]$. В случае $q>2$ будем пользоваться неравенством $d_{l}\left(\tilde{V}, l_{q}^{n}\right) \geq$ $n^{\frac{1}{q}-\frac{1}{2}} d_{l}\left(\tilde{V}, l_{2}^{n}\right)$ и оценивать снизу $d_{l}\left(\tilde{V}, l_{2}^{n}\right)$.

Обозначим $q_{*}=\min \{q, 2\}$. Пусть $\hat{y}=\left(\hat{y}_{j, i}\right)_{1 \leq j \leq s, 1 \leq i \leq \nu_{j}}$,

$$
\hat{y}_{j, i}^{q_{*}^{\prime}}=c_{j, i}^{q_{*}}, \quad \hat{y}_{j, i} \geq 0 .
$$


(При $q \geq 2$ получаем $\hat{y}=\hat{x}$.)

Пусть $L-$ экстремальное подпространство для $d_{l}\left(\tilde{V}, l_{q_{*}}^{n}\right)$ (на нем достигается точная нижняя грань уклонений в определении колмогоровского поперечника), $\operatorname{dim} L=l, Z=L^{\perp}, P-$ ортогональный проектор на $Z$. В силу двойственности [18, с. 101]

$$
\left(d_{l}\left(\tilde{V}, l_{q_{*}}^{n}\right)\right)^{q_{*}^{\prime}} \geq \sup _{z \in Z \cap B_{q_{*}^{\prime}}^{n}} \sup _{x \in \tilde{V}}|\langle z, x\rangle|^{q_{*}^{\prime}} \geq \sup _{g \in G} \sup _{x \in \tilde{V}} \frac{|\langle P g \hat{y}, x\rangle|_{q_{*}^{\prime}}^{q^{\prime}}}{\|P g \hat{y}\|_{q_{*}^{\prime}}^{q_{*}^{\prime}}} \geq \frac{(1 /|G|) \sum_{g \in G}|\langle P g \hat{y}, g \hat{x}\rangle|^{q_{*}^{\prime}}}{(1 /|G|) \sum_{g \in G}\|P g \hat{y}\|_{q_{*}^{*}}^{q_{*}^{*}}} .
$$

Рассмотрим линейные операторы $S: \mathbb{R}^{n} \rightarrow \mathbb{R}^{|G|}, \tilde{S}: \mathbb{R}^{n} \rightarrow \mathbb{R}^{|G|}$, заданные по формулам

$$
(S y)(g)=\langle y, g \hat{x}\rangle, \quad(\tilde{S} y)(g)=\langle y, g \hat{y}\rangle, \quad y \in \mathbb{R}^{n}, g \in G ;
$$

через $\left(S_{g k}\right)_{g \in G, 1 \leq k \leq n}$ и $\left(\tilde{S}_{g k}\right)_{g \in G, 1 \leq k \leq n}$ обозначим их матрицы. Покажем, что

$$
\operatorname{tr}\left(\tilde{S} P S^{*}\right)=\sum_{g \in G}\langle P g \hat{y}, g \hat{x}\rangle .
$$

В самом деле, пусть $T: \mathbb{R}^{|G|} \rightarrow \mathbb{R}^{n}$ - линейный оператор, $\left(T_{k g}\right)_{1 \leq k \leq n, g \in G}$ - его матрица. Тогда $\operatorname{tr}(\tilde{S} T)=\sum_{g \in G} \sum_{k=1}^{n} \tilde{S}_{g k} T_{k g}$. Далее, $\tilde{S}_{g k} \stackrel{(3.10)}{=}\left\langle e_{k}, g \hat{y}\right\rangle$. Пусть $\varphi_{g}-$ вектор в $\mathbb{R}^{|G|}$, у которого на месте $g$ стоит 1 , а на остальных местах нули. Для $T=P S^{*}$ имеем $T_{k g}=\left\langle e_{k}, P S^{*} \varphi_{g}\right\rangle=$ $\left\langle S P e_{k}, \varphi_{g}\right\rangle=\left(S P e_{k}\right)(g) \stackrel{(3.10)}{=}\left\langle P e_{k}, g \hat{x}\right\rangle$. Значит,

$$
\sum_{k=1}^{n} \tilde{S}_{g k} T_{k g}=\sum_{k=1}^{n}\left\langle e_{k}, g \hat{y}\right\rangle\left\langle P e_{k}, g \hat{x}\right\rangle=\sum_{k=1}^{n}\left\langle e_{k}, g \hat{y}\right\rangle\left\langle e_{k}, P g \hat{x}\right\rangle=\langle g \hat{y}, P g \hat{x}\rangle .
$$

Отсюда и из самосопряженности оператора $P$ следует (3.11).

Итак,

$$
\sum_{g \in G}\langle P g \hat{y}, g \hat{x}\rangle=\operatorname{tr}\left(\tilde{S} P S^{*}\right)=\operatorname{tr}\left(S^{*} \tilde{S} P\right) .
$$

Вычислим элементы матрицы $S^{*} \tilde{S}$. Имеем

$$
\left(S^{*} \tilde{S}\right)_{i j}=\left\langle e_{i}, S^{*} \tilde{S} e_{j}\right\rangle=\left\langle S e_{i}, \tilde{S} e_{j}\right\rangle \stackrel{(3.10)}{=} \sum_{g \in G}\left\langle e_{i}, g \hat{x}\right\rangle\left\langle e_{j}, g \hat{y}\right\rangle .
$$

Если $i \neq j$, то эта сумма равна 0 (для каждого $(g \hat{x})_{i}(g \hat{y})_{j} \neq 0$ найдется элемент $\tilde{g} \in G$ такой, что $(\tilde{g} \hat{x})_{i}(\tilde{g} \hat{y})_{j}=-(g \hat{x})_{i}(g \hat{y})_{j}$, причем между $g$ и $\tilde{g}$ можно построить биекцию). Пусть $i=j$, при этом у $e_{i}$ единственная ненулевая координата на номере $(m, t)$. Оценим по порядку сумму $\sum_{g \in G}\left\langle e_{i}, g \hat{x}\right\rangle\left\langle e_{i}, g \hat{y}\right\rangle$. Сначала отметим, что в силу (2.12), (2.23) и условия $\hat{\nu}_{m} / 2 \leq \nu_{m} \leq 2 \hat{\nu}_{m}$ выполнено

$$
\frac{\nu_{m}^{-\theta-1} \varepsilon_{m}}{\sum_{k=1}^{s} \nu_{k}^{-\theta} \varepsilon_{k}} \asymp \frac{n^{-\theta-1}}{\left(\sum_{k=1}^{s} \varepsilon_{k}^{\frac{1}{\theta+1}}\right)^{-\theta-1} \sum_{k=1}^{s} n^{-\theta} \varepsilon_{k}^{\frac{1}{\theta+1}}\left(\sum_{k=1}^{s} \varepsilon_{k}^{\frac{1}{\theta+1}}\right)^{\theta}}=n^{-1} .
$$

Рассмотрим случай $q \geq 2$. Напомним, что тогда $\hat{y}=\hat{x}$. Имеем

$$
\begin{gathered}
\sum_{g \in G}\left\langle g \hat{x}, e_{i}\right\rangle^{2}=\frac{|G|}{\left|G_{m}\right|} \sum_{g_{m} \in G_{m}}\left\langle g_{m} \hat{x}, e_{i}\right\rangle^{2}=\frac{|G|}{\left|G_{m}\right|}\left(\nu_{m}-1\right) ! 2^{\nu_{m}} \sum_{k=1}^{\nu_{m}} c_{m, k}^{2} \\
=\frac{|G|}{\nu_{m}} \sum_{k=1}^{\nu_{m}} c_{m, k}^{2}\left(\underset{p, 4),(3.6),(3.7)}{\overparen{|G|}} \frac{|G|}{\nu_{m}} A \frac{\nu_{m}^{-\theta} \varepsilon_{m}}{\sum_{k=1}^{s} \nu_{k}^{-\theta} \varepsilon_{k}} \underset{\widehat{p}, q}{(3.13)} A n^{-1}|G| .\right.
\end{gathered}
$$


Учитывая (3.6), получаем, что матрица $S^{*} \tilde{S}$ пропорциональна единичной. Значит,

$$
\frac{1}{|G|} \sum_{g \in G}\langle P g \hat{y}, g \hat{x}\rangle \underset{\overrightarrow{p, q}}{(3.12)} A n^{-1} \operatorname{tr} P \asymp A
$$

(так как $\operatorname{dim} \operatorname{Im} P \geq n / 2$ ). В силу неравенства Гельдера получаем оценку снизу для числителя в (3.9):

$$
\frac{1}{|G|} \sum_{g \in G}|\langle P g \hat{y}, g \hat{x}\rangle|^{2} \geq\left(\frac{1}{|G|} \sum_{g \in G}\langle P g \hat{y}, g \hat{x}\rangle\right)^{2} \underset{p, q}{\asymp} A^{2} .
$$

Пусть теперь $q \leq 2$. Тогда

$$
\begin{gathered}
\sum_{g \in G}\left\langle g \hat{x}, e_{i}\right\rangle\left\langle g \hat{y}, e_{i}\right\rangle=\frac{|G|}{\left|G_{m}\right|} \sum_{g_{m} \in G_{m}}\left\langle g_{m} \hat{x}, e_{i}\right\rangle\left\langle g_{m} \hat{y}, e_{i}\right\rangle \stackrel{(3.8)}{=} \frac{|G|}{\left|G_{m}\right|}\left(\nu_{m}-1\right) ! 2^{\nu_{m}} \sum_{k=1}^{\nu_{m}}\left|c_{m, k}\right|^{q} \\
=\frac{|G|}{\nu_{m}} \sum_{k=1}^{\nu_{m}}\left|c_{m, k}\right|^{(3.5)-(3.7)} \underset{\widetilde{p, q}}{\widetilde{|G|}} \frac{\mid \nu_{m}^{-\theta} \varepsilon_{m}}{\sum_{m}^{s} \nu_{k}^{-\theta} \varepsilon_{k}} \underset{\hat{p, q}}{(3.13)} A n^{-1}|G| .
\end{gathered}
$$

Учитывая (3.6), снова получаем (3.14); в силу неравенства Гельдера, для числителя в (3.9) выполнена оценка $(1 /|G|) \sum_{g \in G}|\langle P g \hat{y}, g \hat{x}\rangle|^{q_{*}^{\prime}} \underset{p, q}{\gtrsim} A^{q^{\prime}}$.

Теперь получим оценку сверху для знаменателя в (3.9). Имеем

$$
\frac{1}{|G|} \sum_{g \in G}\|P g \hat{y}\|_{q_{*}^{\prime}}^{q_{*}^{\prime}}=\sum_{i=1}^{n} \frac{1}{|G|} \sum_{g \in G}\left|\left\langle g \hat{y}, P e_{i}\right\rangle\right|^{q_{*}^{\prime}} .
$$

Далее рассуждаем так же, как в лемме из [9, с. 37-38]. Оценим сверху наименьшую константу в неравенстве

$$
\left(\frac{1}{|G|} \sum_{g \in G}|\langle g \hat{y}, y\rangle|^{q_{*}^{\prime}}\right)^{1 / q_{*}^{\prime}} \leq C\|y\|_{2} .
$$

Из неравенства Хинчина

$$
\frac{1}{2^{n}} \sum_{\delta_{k}= \pm 1}\left|\sum_{k=1}^{n} \delta_{k} a_{k}\right|^{q_{*}^{\prime}} \underset{q_{*}}{\lesssim}\left(\sum_{k=1}^{n} a_{k}^{2}\right)^{q_{*}^{\prime} / 2}
$$

(см., например, [19, с. 178]) выводится соотношение

$$
\frac{1}{|G|} \sum_{g \in G}|\langle g \hat{y}, y\rangle|^{q_{*}^{\prime}} \underset{q_{*}}{\lesssim} \frac{1}{|G|} \sum_{g \in G}\left(\sum_{1 \leq i \leq n}\left|y_{i}(g \hat{y})_{i}\right|^{2}\right)^{q_{*}^{\prime} / 2} .
$$

Поэтому достаточно получить оценку на константу в неравенстве

$$
\frac{1}{|G|} \sum_{g \in G}\left(\sum_{1 \leq i \leq n}\left|y_{i}(g \hat{y})_{i}\right|^{2}\right)^{q_{*}^{\prime} / 2} \leq C^{q_{*}^{\prime}}\|y\|_{2}^{q_{*}^{\prime}}
$$

Пользуясь соображениями выпуклости (здесь учитывается условие $q_{*} \leq 2$ ), получаем, что достаточно подставить $y=e_{i}$. Если у $e_{i}$ единственная ненулевая координата находится на месте $(j, t)$, то

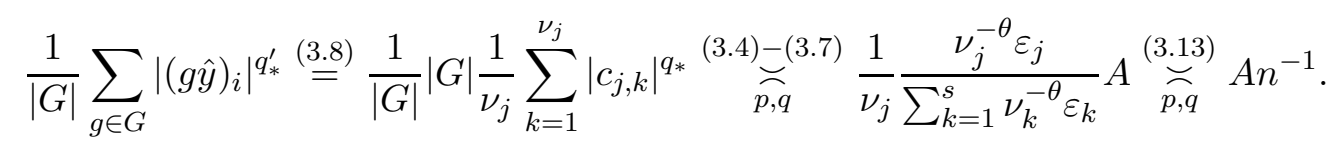


Значит, $C^{q_{*}^{\prime}} \underset{p, q}{\lesssim} A n^{-1}$. Отсюда получаем

$$
\sum_{i=1}^{n} \frac{1}{|G|} \sum_{g \in G}\left|\left\langle g \hat{y}, P e_{i}\right\rangle\right|^{q_{*}^{\prime}} \underset{p, q}{\lesssim} C^{q_{*}^{\prime}} \sum_{i=1}^{n}\left\|P e_{i}\right\|_{2}^{q_{*}^{\prime}} \leq C^{q_{*}^{\prime}} n \underset{p, q}{\lesssim} A .
$$

Поэтому $\left(d_{l}\left(V, l_{q_{*}}^{n}\right)\right)^{q_{*}^{\prime}} \underset{p, q}{\stackrel{(3.9)}{\gtrsim}} \frac{A^{q_{*}^{\prime}}}{A}=A^{q_{*}^{\prime}-1}$, т. е.

$$
d_{l}\left(V, l_{q_{*}}^{n}\right) \underset{p, q}{\gtrsim} A^{\frac{q_{*}^{\prime}-1}{q_{*}^{\prime}}}=A^{1 / q_{*}}
$$

что дает правильную по порядку оценку снизу.

Случай $p \leq q, p \leq 2$. Возьмем $\nu_{j} \asymp n / s$. Достаточно получить оценку снизу для множества $V$ последовательностей $\left(c_{j, i}\right)_{1 \leq j \leq s, 1 \leq i \leq \nu_{j}}$, удовлетворяющих ограничениям

$$
\sum_{j=1}^{s} n^{\left(1+\frac{1}{q}-\frac{1}{p}\right) p} \sum_{i=1}^{\nu_{j}}\left|c_{j, i}\right|^{p} \leq 1, \quad \sum_{i=1}^{\nu_{j}}\left|c_{j, i}\right| \leq \frac{\varepsilon_{j}}{s} \frac{1}{n^{1 / q}}, \quad 1 \leq j \leq s
$$

(см. (1.11)). Если

$$
\sum_{j=1}^{s} \sum_{i=1}^{\nu_{j}}\left|c_{j, i}\right| \leq n^{-1-\frac{1}{q}+\frac{1}{p}}
$$

то для любого $1 \leq j \leq s$ выполнено $\sum_{i=1}^{\nu_{j}}\left|c_{j, i}\right| \leq n^{-1-\frac{1}{q}+\frac{1}{p}}$, и поэтому при достаточно больших $n($ так как $p>1)$

$$
\sum_{i=1}^{\nu_{j}}\left|c_{j, i}\right| \leq \frac{\varepsilon_{j}}{s n^{1 / q}}
$$

Значит,

$$
V \supset c(p, q) n^{-1-\frac{1}{q}+\frac{1}{p}} B_{1}^{n}
$$

для некоторого $c(p, q)>0$. Остается применить оценки для $d_{l}\left(n^{-1-\frac{1}{q}+\frac{1}{p}} B_{1}^{n}, l_{q}^{n}\right)$, полученные в $[15 ; 17]$.

Поперечники множества $V$, возникающего в задаче об оценке $d_{n}\left(\tilde{M}, L_{q}[0,1]\right)$, оцениваются снизу аналогичным образом. В качестве группы $G$ берется

$$
\prod_{j=1}^{s} G_{j}, \quad G_{j}=\{ \pm 1\}^{4 \nu_{j}} \times \tilde{S}_{4 \nu_{j}}
$$

где $\tilde{S}_{4 \nu_{j}}$ - множество перестановок вида $4 k+i \mapsto 4 \pi(k)+i, \pi \in S_{\nu_{j}}, 0 \leq k \leq \nu_{j}-1,1 \leq i \leq 4$.

\section{СПИСОК ЛИТЕРАТУРЫ}

1. Васильева А.А. Критерий существования гладкой функции при ограничениях // Мат. заметки. 2007. T. 82 , № 3. C. 335-346.

2. Тихомиров В.М. Некоторые вопросы теории приближений. М.: Изд-во МГУ, 1976. 304 р.

3. Тихомиров B.M. Теория приближений // Современные проблемы математики. Фундаментальные направления. Т. 14. (Итоги науки и техники. ВИНИТИ АН СССР). Москва, 1987. С. 103-260.

4. Pinkus A. $n$-widths in approximation theory. Berlin: Springer, 1985. 294 p. doi: 10.1007/978-3-64269894-1.

5. Галеев Э.М. Поперечники по Колмогорову пересечения классов периодических функций и конечномерных множеств // Мат. заметки. 1981. Т. 29, № 5. С. 749-760. 
6. Галеев Э.М. Поперечники по Колмогорову некоторых конечномерных множеств в смешанной норме. // Мат. заметки. 1995. Т. 58, № 1. С. 144-148.

7. Галеев Э.М. Поперечники по Колмогорову классов периодических функций одной и нескольких переменных // Изв. АН СССР. Сер. математическая. 1990. Т. 54, № 2. С. 418-430.

8. Галеев Э.М. Поперечники функциональных классов и конечномерных множеств // Владикавказский мат. журн. 2011. Т. 13, № 2. С. 3-14.

9. Глускин Е.Д. Пересечения куба с октаэдром плохо аппроксимируются подпространствами малой размерности // Приближение функций специальными классами операторов: межвуз. сб. научн. тр. / Мин. прос. РСФСР; Вологодский гос. пед. ин-т. Вологда, 1987. С. 35-41.

10. Изаак А.Д. Поперечники по Колмогорову в конечномерных пространствах со смешанной нормой // Мат. заметки. 1994. Т. 55, № 1. С. 43-52.

11. Изаак А.Д. Поперечники классов Гёльдера-Никольского и конечномерных множеств в пространствах со смешанной нормой // Мат. заметки. 1996. Т. 59, № 3. С. 459-461.

12. Малыхин Ю.В., Рютин К.С. Произведение октаэдров плохо приближается в метрике $l_{2,1} / /$ Мат. заметки. 2017. Т. 101, № 1. С. 85-90.

13. Исмагилов Р.С. Поперечники множеств в линейных нормированных пространствах и приближение тригонометрическими многочленами // Успехи мат. наук. 1974. Т. 29, №3. С. 161-178.

14. Кашин Б.С. Поперечники некоторых конечномерных множеств и классов гладких функций // Изв. АН СССР. Сер. математическая. 1977. Т. 41, №2. С. 334-351.

15. Глускин Е.Д. Нормы случайных матриц и поперечники конечномерных множеств // Мат. сборник. 1983. Т. 120 (162), №. 2. С. 180-189.

16. Майоров В.Е. Дискретизация задачи о поперечниках // Успехи мат. наук. 1975. Т. 30 , № 6. C. $179-180$.

17. Глускин Е.Д. О некоторых конечномерных задачах теории поперечников // Вестн. ЛГУ. 1981. Т. 13. С. $5-10$.

18. Иоффе А.Д., Тихомиров В.М. Двойственность выпуклых функций и экстремальные задачи // Успехи мат. наук. 1968. Т. 23, № 6. С. 51-116.

19. Fabian M., Habala P., Hájek P., Montesinos Santalucia V., Pelant J., Zizler V. Functional analysis and infinite-dimensional geometry. N Y: Springer, 2001. 451 p. (Ser. CMS Books in Math.) doi: 10.1007/978-1-4757-3480-5 .

Поступила 15.03.2019

После доработки 17.05.2019

Принята к публикации 20.05.2019

Васильева Анастасия Андреевна

д-р физ.-мат. наук, доцент

Московский государственный университет им. М. В. Ломоносова,

г. Москва

e-mail: vasilyeva_nastya@inbox.ru

\section{REFERENCES}

1. Vasil'eva A.A. An existence criterion for a smooth function under constraints. Math Notes, 2007, vol. 82, no. 3-4, pp. 295-308. doi: 10.1134/S0001434607090027.

2. Tikhomirov V.M. Nekotorye voprosy teorii priblizhenii [Some questions in approximation theory]. Moscow: Izdat. Moskov. Gos. Univ., 1976, 304 p.

3. Tikhomirov V.M. Theory of approximations. Itogi Nauki Tekh., Ser. Sovrem. Probl. Mat., Fundam. Napravleniya, 1987, vol. 14, pp. 103-260.

4. Pinkus A. $n$-widths in approximation theory. Berlin: Springer, 1985, $294 \mathrm{p}$. doi: 10.1007/978-3-642-69894-1.

5. Galeev E.M. The Kolmogorov diameter of the intersection of classes of periodic functions and of finitedimensional sets. Math. Notes, 1981, vol. 29, no. 5, pp. 382-388. doi: 10.1007/BF01158363.

6. Galeev E.M. Kolmogorov $n$-width of some finite-dimensional sets in a mixed measure. Math. Notes, 1995, vol. 58, no. 1, pp. 774-778. doi: 10.1007/BF02306188. 
7. Galeev E.M. Kolmogorov widths of classes of periodic functions of one and several variables. Math. USSR-Izv., 1991, vol. 36, no. 2, pp. 435-448. doi: 10.1070/IM1991v036n02ABEH002029 .

8. Galeev E.M. Kolmogorov and linear diameters of functional classes and finite dimensional sets. Vladikavkaz. Mat. Zh., 2011, vol. 13, no. 2, pp. 3-14 (in Russian).

9. Gluskin E.D. Intersections of a cube with an octahedron are poorly approximated by subspaces of small dimension. Approximation of functions by special classes of operators, Interuniv. Collect. Sci. Works, Vologda, 1987, pp. 35-41 (in Russian).

10. Izaak A.D. Kolmogorov widths in finite-dimensional spaces with mixed norms. Math. Notes, 1994, vol. 55, no. 1, pp. 30-36. doi: 10.1007/BF02110761.

11. Izaak A.D. Widths of Hölder-Nikol'skij classes and finite-dimensional subsets in spaces with mixed norm. Math. Notes, 1996, vol. 59, no. 3, pp. 328-330. doi: 10.1007/BF02308549.

12. Malykhin Yu.V., Ryutin K.S. The product of octahedra is badly approximated in the $\ell_{2,1}$-metric. Math. Notes, 2017, vol. 101, no. 1, pp. 94-99. doi: 10.1134/S0001434617010096.

13. Ismagilov R.S. Diameters of sets in normed linear spaces and the approximation of functions by trigonometric polynomials. Russian Math. Surveys, 1974, vol. 29, no. 3, pp. 169-186. doi: 10.1070/RM1974v029n03ABEH001287.

14. Kashin B.S. Diameters of some finite-dimensional sets and classes of smooth functions. Math. USSR-Izv., 1977, vol. 11, no. 2, pp. 317-333. doi: 10.1070/IM1977v011n02ABEH001719.

15. Gluskin E.D. Norms of random matrices and widths of finite-dimensional sets. Math. USSR-Sb., 1984, vol. 48, no. 1, pp. 173-182. doi: 10.1070/SM1984v048n01ABEH002667.

16. Maiorov V.E. Discretization of the problem of diameters. Uspekhi Mat. Nauk, 1975, vol. 30, no. 6 (186), pp. 179-180 (in Russian).

17. Gluskin E.D. On some finite-dimensional problems of the theory of diameters. Vestn. Leningr. Univ., 1981, vol. 13, no. 3, pp. 5-10 (in Russian).

18. Ioffe A.D., Tikhomirov V.M. Duality of convex functions and extremum problems. Russian Math. Surveys, 1968, vol. 23, no. 6, pp. 53-124. doi: 10.1070/RM1968v023n06ABEH001251.

19. Fabian M., Habala P., Hájek P., Montesinos Santalucia V., Pelant J., Zizler V. Functional analysis and infinite-dimensional geometry. Ser. CMS Books in Math. N Y: Springer, 2001, 451 p. doi: $10.1007 / 978-1-4757-3480-5$.

Received March 15, 2019

Revised May 17, 2019

Accepted May 20, 2019

Funding Agency: This work was supported by the Russian Foundation for Basic Research (project no. 19-01-00332).

Anastasia Andreevna Vasil'eva, Dr. Phys.-Math. Sci., Assoc. Prof., Lomonosov Moscow State University, faculty on mechanics and mathematics, Moscow, Vorobyovy gory, 1, Main Building of MSU, 119991 Russia, e-mail: vasilyeva_nastya@inbox.ru.

Cite this article as: A. A. Vasil'eva. Kolmogorov widths of Sobolev classes on a closed interval with constraints on the variation, Trudy Instituta Matematiki i Mekhaniki URO RAN, 2019, vol. 25, no. 2 , pp. $48-66$. 